\title{
Burned to the Bone
}

AMÉLIE BOHM-SIGRAND, MD, Department of Rheumatology, François-Mitterrand Teaching Hospital; PIERRE POTTECHER, MD, PhD, Department of Radiology, Section of Musculoskeletal Imaging and Intervention, LE2I UMR CNRS 6306, University of Burgundy, François-Mitterrand Teaching Hospital; PAUL ORNETTI, MD, PhD, Department of Rheumatology, INSERM 1093, University of Burgundy, François-Mitterrand Teaching Hospital, Dijon Cedex, France. Address correspondence to Pr. P. Ornetti, Department of Rheumatology, 14 rue Gaffarel, CHU dijon, 21000 Dijon, France. E-mail: paul.ornetti@chu-dijon.fr. Ethical approval was not required in accordance with the policy of our institution and the type of manuscript (case report). We have obtained the patient's written informed consent to publish the material. J Rheumatol 2017;44:1507-8; doi:10.3899/jrheum.161388

A burn-related injury that had worsened over time was compatible upon radioclinical examination with severe psoriatic arthritis (PsA), but was missing several essential characteristics of that disorder.

A 58-year-old woman with no personal or family history of rheumatic diseases consulted for hand arthralgia. Her medical history was marked by severe burns to $80 \%$ of her body, sparing the face and the back, following a gas explosion 20 years earlier. Initially, systematic excision and auto-grafting of third-degree burns to the hand and feet was performed without arthrodesis or local septic complications, followed by intensive physical therapy.

Clinical examination showed soft tissue loss, skin contracture, and finger deformation (Figure 1), which had gradually worsened over time [swan-neck deformities, a boutonniere deformity (white arrows) associated with hyperkeratosis or onycholysis of certain nails]. Radiography (Figure 2) showed marked joint space narrowing of distal
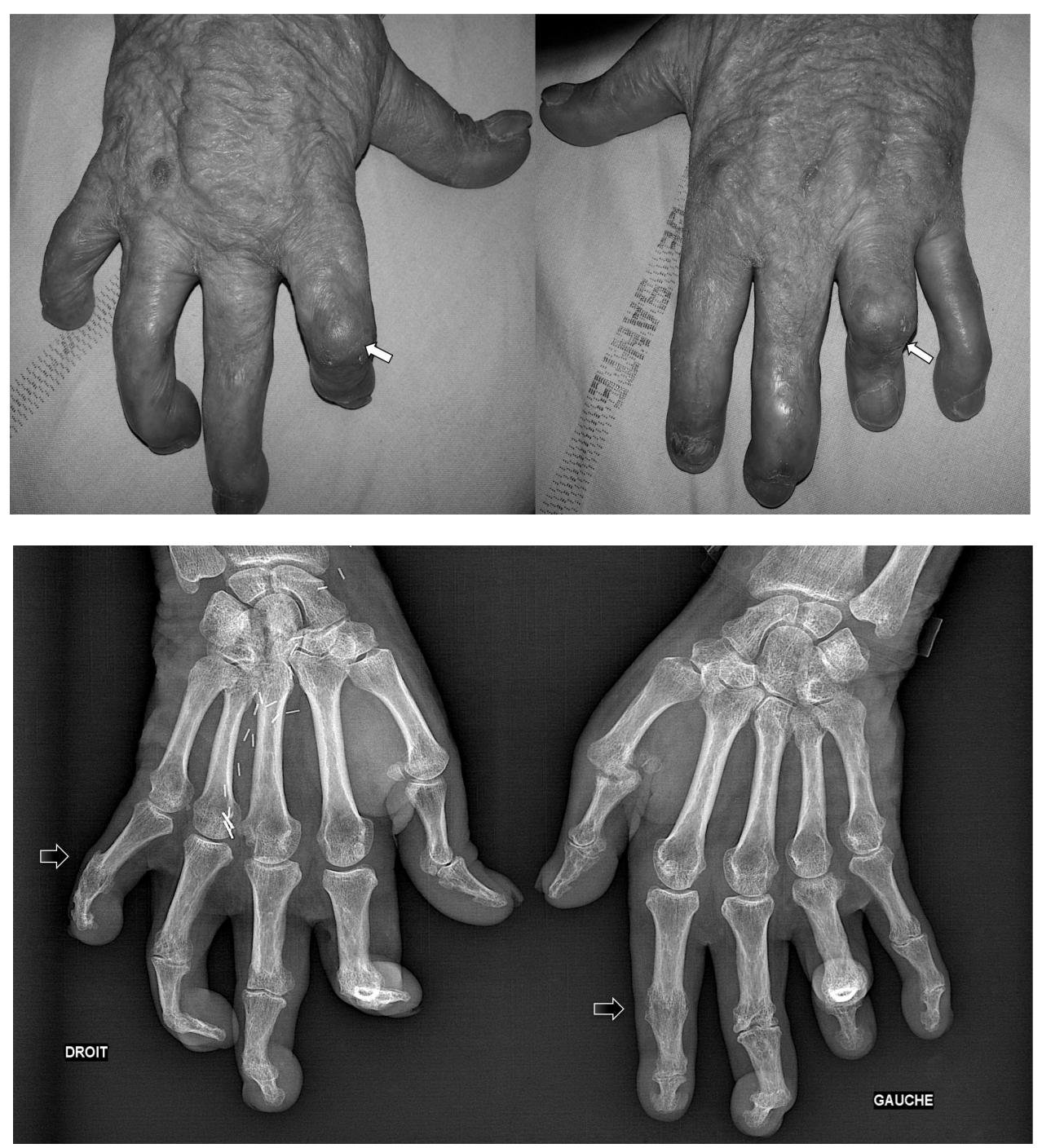

Figure 1. Polyarthralgia affecting the hands with severe deformity of proximal and interphalangeal joints and chronic onychopathy.
Figure 2. Hand radiograph showing joint space narrowing of distal interphalangeal joints and bony ankylosis of certain proximal interphalangeal joints.

Personal non-commercial use only. The Journal of Rheumatology Copyright ( 2017. All rights reserved. 
interphalangeal joints, bony ankylosis of certain proximal interphalangeal joints (black arrows), and subluxation of the metacarpophalangeal joint of the left thumb. Knee radiographs showed bilateral heterotopic ossification. The radioclinical examination was compatible with severe PsA characterized by both destructive arthritis and psoriatic onychopathy. However, the absence of HLA-B27, the absence of synovial or entheseal inflammation on magnetic resonance imaging, serial normal C-reactive protein levels, and the lack of efficacy of antiinflammatory drugs ruled out this hypothesis.

A diagnosis of burn-related injury ${ }^{1,2}$ characterized by joint destruction, bony ankyloses, and nail abnormalities was made and only longterm rehabilitation and splinting were proposed to improve functionality of the hands.

This rare differential diagnosis of PsA must be kept in mind to avoid treatment with disease-modifying antirheumatic drugs, which could be dangerous given the high risk of infection in burn patients.

\section{REFERENCES}

1. Davis DL, Resnik CS. Case 229: Burn-related global ankylosis of interphalangeal joints with associated acroosteolysis. Radiology 2016;279:645-9.

2. Pandit SK, Malla CN, Zarger HU, Kaul A, Dev G. A study of bone and joint changes secondary to burns. Burns 1993;19:227-8. 